\title{
Detection of viral RNA in diverse body fluids in an SFTS patient with encephalopathy, gastrointestinal bleeding and pneumonia: a case report and literature review
}

Kazumasa Akagi ${ }^{1,2}$, Taiga Miyazaki ${ }^{1,3^{*}}$, Kazuhiro Oshima ${ }^{1,4}$, Asuka Umemura ${ }^{1,5}$, Satoshi Shimada ${ }^{6}$, Kouichi Morita $^{6}$, Hiroaki Senju1, Masato Tashiro ${ }^{3,7}$, Takahiro Takazono 1,3, Tomomi Saijo ${ }^{1}$, Shintaro Kurihara ${ }^{7}$, Motohiro Sekino ${ }^{8}$, Kazuko Yamamoto', Yoshifumi Imamura', Koichi Izumikawa ${ }^{3,7}$, Katsunori Yanagihara ${ }^{9}$, Akihiko Uda ${ }^{10}$, Shigeru Morikawa ${ }^{10}$, Tomoki Yoshikawa ${ }^{11}$, Takeshi Kurosu ${ }^{11}$, Masayuki Shimojima ${ }^{11}$, Masayuki Saijo ${ }^{11}$ and Hiroshi Mukae ${ }^{1}$

\begin{abstract}
Background: Severe fever with thrombocytopenia syndrome (SFTS) is an emerging infectious disease that commonly has a lethal course caused by the tick-borne Huaiyangshan banyang virus [former SFTS virus (SFTSV)]. The viral load in various body fluids in SFTS patients and the best infection control measure for SFTS patients have not been fully established.

Case presentation: A 79-year-old man was bitten by a tick while working in the bamboo grove in Nagasaki Prefecture in the southwest part of Japan. Due to the occurrence of impaired consciousness, he was referred to Nagasaki University Hospital for treatment. The serum sample tested positive for SFTSV-RNA in the genome amplification assay, and he was diagnosed with SFTS. Furthermore, SFTSV-RNA was detected from the tick that had bitten the patient. He was treated with multimodal therapy, including platelet transfusion, antimicrobials, antifungals, steroids, and continuous hemodiafiltration. His respiration was assisted with mechanical ventilation. On day 5, taking the day on which he was hospitalized as day 0, serum SFTSV-RNA levels reached a peak and then decreased. However, the cerebrospinal fluid collected on day 13 was positive for SFTSV-RNA. In addition, although serum SFTSV-RNA levels decreased below the detectable level on day 16, he was diagnosed with pneumonia with computed tomography. SFTSV-RNA was detected in the bronchoalveolar lavage fluid on day 21. By day 31, he recovered consciousness completely. The pneumonia improved by day 51, but SFTSV-RNA in the sputum remained (Continued on next page)
\end{abstract}

\footnotetext{
* Correspondence: taiga-m@nagasaki-u.ac.jp

'Department of Respiratory Medicine, Nagasaki University Hospital, Nagasaki, Japan

${ }^{3}$ Department of Infectious Diseases, Nagasaki University Graduate School of Biomedical Sciences, Nagasaki, Japan

Full list of author information is available at the end of the article
}

(C) The Author(s). 2020 Open Access This article is licensed under a Creative Commons Attribution 4.0 International License, which permits use, sharing, adaptation, distribution and reproduction in any medium or format, as long as you give appropriate credit to the original author(s) and the source, provide a link to the Creative Commons licence, and indicate if changes were made. The images or other third party material in this article are included in the article's Creative Commons licence, unless indicated otherwise in a credit line to the material. If material is not included in the article's Creative Commons licence and your intended use is not permitted by statutory regulation or exceeds the permitted use, you will need to obtain permission directly from the copyright holder. To view a copy of this licence, visit http://creativecommons.org/licenses/by/4.0/ The Creative Commons Public Domain Dedication waiver (http://creativecommons.org/publicdomain/zero/1.0/) applies to the data made available in this article, unless otherwise stated in a credit line to the data. 
(Continued from previous page)

positive for approximately 4 months after disease onset. Strict countermeasures against droplet/contact infection were continuously conducted.

Conclusions: Even when SFTSV genome levels become undetectable in the serum of SFTS patients in the convalescent phase, the virus genome remains in body fluids and tissues. It may be possible that body fluids such as respiratory excretions become a source of infection to others; thus, careful infection control management is needed.

Keywords: SFTS, Viremia, Encephalopathy, Pneumonia, Case report

\section{Background}

Severe fever with thrombocytopenia syndrome (SFTS), an infectious disease caused by the tick-borne Huaiyangshan banyangvirus [former SFTS virus (SFTSV)], Banyangvirus [former Phlebovirus] genus, Phenuiviridae (former Buniyaviridae) family [1]. SFTS is a viral hemorrhagic fever with a high case fatality rate. Patients with SFTS show symptoms such as a sudden fever, vomiting, diarrhea, stomachache, and general fatigue in the early phase of the disease, followed by hemorrhagic symptoms and unconsciousness in severe cases [2, 3]. Thrombocytopenia, leukopenia and elevation in liver enzymes are commonly demonstrated in blood examination. In Japan, the mortality rate of patients with SFTS is approximately $30 \%$ [4].

Since SFTSV infection in humans in China was reported in 2009, cases of SFTS have been reported in China, South Korea, and Japan [5-7]. While much progress toward understanding the pathophysiology and clinical characteristics of this disease has been made in recent years $[3,8]$, limited information is available about the period of viral excretion associated with complications of SFTS. Here, we report a patient with SFTS who developed viremia, encephalopathy and pneumonia and was successively treated with multimodal therapies. The SFTSV genome level was checked in the late stage of the disease in serum, cerebrospinal fluid (CSF), and sputum of the patient.

\section{Case presentation}

\section{Patient}

The patient was a 79-year-old Japanese man who lived in the southwest part of Japan. He had been undergoing hemodialysis for 4 years due to end-stage diabetic nephropathy. He had been working in a bamboo grove surrounding his house for 4 days just before admission (day -4 to day -1$)$. Lightheadedness occurred on day -1 , and the next day, he visited a local clinic (day 0). A blood test showed thrombocytopenia [platelet count of $71,000 / \mu \mathrm{L}$ (normal range 158,000-348,000/ $\mu \mathrm{L}$ )] and elevated liver enzymes [aspartate transaminase of $287 \mathrm{U} / \mathrm{L}$ (normal range 13-30 U/L) and alanine transaminase of $139 \mathrm{U} / \mathrm{L}$ (normal range 10-42 U/L)]. Impaired consciousness occurred, and he was then referred to and admitted to Nagasaki University Hospital on the same day (day 0).

\section{Physical findings on admission day}

On admission (day 0), the patient showed drowsiness with a Japan Coma Scale of II-20 and a Glasgow Coma Scale of 14/15 (E3V5M6). He had a body temperature of $38.4{ }^{\circ} \mathrm{C}$, blood pressure of $141 / 75 \mathrm{mmHg}$, pulse rate of 84 beats $/ \mathrm{min}$, and respiratory rate of 16 breaths $/ \mathrm{min}$. Oxygen saturation was $92 \%$ on room air. A swollen tick by blood sucking was attached on the surface of the right precordium (Fig. 1). Palpable cervical and inguinal lymph nodes were found on the right side. He had

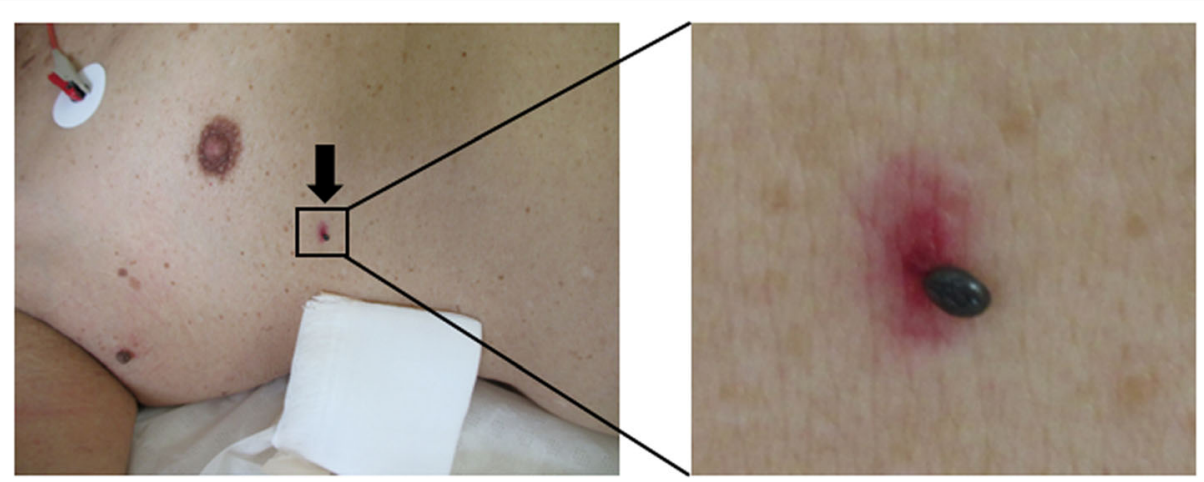

Fig. 1 A swollen tick biting the right precordium of the patient (black arrow). SFTSV-RNA was detected from this tick and the patient 
multiple unraised red spots on the left thigh and erythema associated with exfoliation on the groin of both sides. His chest/abdomen examination did not reveal any abnormal findings. Neck stiffness was not apparent.

\section{Laboratory and imaging findings on admission day}

Blood test results from the first examination are shown in Additional file 1. The hematology test showed pancytopenia, with a white blood cell count of $1800 / \mu \mathrm{L}$ (normal range 3300-8600/ $\mathrm{LL}$ ), hemoglobin of $10.9 \mathrm{~g} / \mathrm{dL}$ (normal range 13.7-16.8 g/dL), and platelet count of 52, $000 / \mu \mathrm{L}$. Biochemistry tests showed elevation in liver enzymes [aspartate transaminase of $347 \mathrm{U} / \mathrm{L}$, alanine transaminase of $151 \mathrm{U} / \mathrm{L}, \gamma$-glutamyltransferase of $109 \mathrm{U} / \mathrm{L}$ (normal range 13-64 U/L), and lactate dehydrogenase of $878 \mathrm{U} / \mathrm{L}$ (normal range 124-222 U/L)]. The serum levels of ferritin and soluble interleukin-2 receptor were 2627 $\mathrm{ng} / \mathrm{mL}$ (normal range $40-465 \mathrm{ng} / \mathrm{mL}$ ) and $2135 \mathrm{U} / \mathrm{mL}$ (normal range 127-582 U/mL), respectively. Hemophagocytosis was observed in bone marrow aspirates. Blood culture revealed no microorganisms. Right axillary lymphadenopathy was observed in a chest and abdominal radiograph/contrast-enhanced computed tomography $(\mathrm{CT})$ scan. There were no particularly abnormal findings in a noncontrast head CT scan, electrocardiogram, or echocardiograph.

\section{Clinical course}

On admission (day 0), differential diagnoses included SFTS and rickettsial infections. In accordance with the treatment for severe rickettsial infections, we started infusion of minocycline $(200 \mathrm{mg} /$ day $)$ and levofloxacin (500 mg/day) combined with platelet transfusion and recombinant human thrombomodulin administration (8320 U/day) (Fig. 2). Intravenous immunoglobulin was given as an adjunct therapy. On day 1 , unconsciousness rapidly progressed, and a generalized seizure with respiratory failure occurred. His respiration was supported with mechanical ventilation, and systemic management, including continuous hemodiafiltration, was initiated in an intensive care unit. Administration of meropenem (3 g/day) was also started. On day 2 , a real-time quantitative reverse transcription-polymerase chain reaction (qRT-PCR) assay for SFTSV, which was performed as described previously [9], was positive (5.97 $\log _{10}$ copies/ $\mathrm{mL}$ ) in the serum. Furthermore, qRT-PCR for the tick that had bitten the patient was positive (see Additional file 2). No Rickettsiaceae, including Orientia tsutsugamushi and Rickettsia japonica, was detected by duplex real-time PCR [10] using blood and eschar samples.

We administered $200 \mathrm{mg} /$ day hydrocortisone from day 2 considering the possibility of relative adrenal insufficiency. The levels of liver enzymes significantly increased on day 4 , and minocycline, levofloxacin and immunoglobulin preparations were discontinued. Because $\beta$-D glucan levels increased at $131.1 \mathrm{pg} / \mathrm{mL}$ (MK-II assay; negative cutoff value $\leq 20 \mathrm{pg} / \mathrm{mL}$ ) and both serum Aspergillus and Candida antigen were positive with a value of 4.6 for Aspergillus antigen (negative cutoff index $<0.5$ ), the central venous catheter was replaced, and presumptive therapy with caspofungin $70 \mathrm{mg} /$ day was initiated (Fig. 2). After that, serum Cryptococcus antigen was positive (titer; 1:1), and considering the possibility of trichosporonosis, voriconazole $280 \mathrm{mg} /$ day was added to the treatment regimen. On day 5, the serum SFTSV-RNA level reached a peak $\left(9.31 \log _{10}\right.$ copies $\left./ \mathrm{mL}\right)$ and then decreased. On day 6, severe melena appeared. Lower gastrointestinal endoscopy showed mucous and bloody stool, intestinal edema, and severe rectal hemorrhage. A stool culture detected Candida glabrata. On day $8, \beta$-D glucan levels further increased to $425.5 \mathrm{pg} / \mathrm{mL}$, and a blood culture detected Candida glabrata; therefore, we judged that bacterial translocation occurred from the intestine. Caspofungin and voriconazole were changed to liposomal amphotericin B $250 \mathrm{mg} /$ day, and meropenem was changed to the combination therapy with tazobactam/piperacillin and vancomycin. The levels of liver enzymes peaked on day 8 , after which they tended to decrease. After day 12, platelet transfusion became unnecessary. Because impaired consciousness continued even after the sedative was discontinued, encephalopathy was suspected. A lumbar puncture was performed on day 13, and CSF was obtained. The qRT-PCR of CSF to detect SFTSV was positive with a value of $4.10 \log _{10}$ copies/ $\mathrm{mL}$. On day 16, the serum SFTSV-RNA level dropped below the detectable level. On day 31, the patient was discharged from the intensive care unit, and the level of consciousness improved approximately one month after disease onset. There was a swollen right axillary lymph node at the first examination, which remained swollen after viruses disappeared from the blood (Fig. 3). However, we could not judge whether SFTSV remained in the lymph node because we did not perform a lymph node biopsy.

Although a chest CT scan on admission did not show clear pneumonia findings, infiltrative shadows on the basal area of both lungs were found by a chest CT scan on day 9 (Fig. 3). In bronchoalveolar lavage fluid (BALF) obtained on day 21, neutrophils were predominant in the cellular fraction, but general types of bacteria were not detected, probably because broadspectrum antimicrobials had already been administered. However, Cryptococcus antigen (titer; 1:2), Aspergillus antigen (a value of 8.6), and SFTSV-RNA (2.51 $\log _{10}$ copies $/ \mathrm{mL}$ ) were detected, and Aspergillus niger was cultured from the BALF. Therefore, we speculated that 


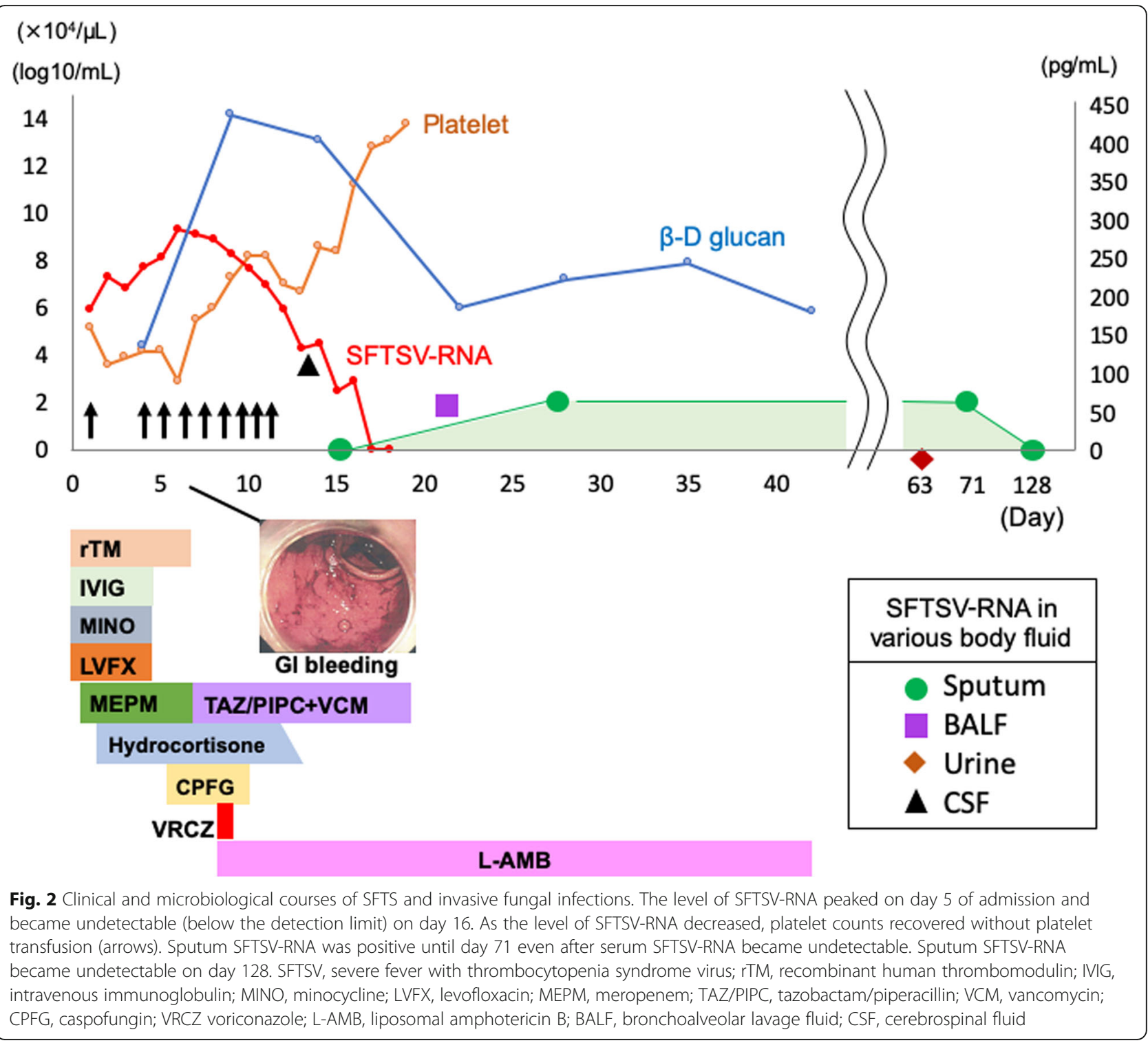

fungal pneumonia was induced by transient immunosuppression caused by SFTSV infection and continued liposomal amphotericin B administration. A chest CT scan on day 30 showed that infiltrative shadows were organized overall. Liposomal amphotericin B and tazobactam/piperacillin were discontinued on days 37 and 42, respectively. A slight ground-glass opacity remained, but infiltrative shadows improved on day 51 . Although $\beta$-D glucan levels remained high and Aspergillus antigen was persistently positive, there was no relapse of aspergillosis or candidemia. SFTSV-RNA was persistently detected in the sputum until day 71 despite its disappearance in the blood on day 16. After confirming that SFTSV-RNA became negative in the sputum on day 127, we stopped performing droplet isolation precautions on the patient.

\section{Discussion and conclusions}

A meta-analysis on risk factors for mortality in patients with SFTS has shown advanced age; high viremia levels; low albumin levels; low platelet count; high aspartate transaminase, alanine transaminase, lactate dehydrogenase, and creatine kinase levels; and prolonged activated partial thromboplastin time [11]. The patient met all of the above factors, and his disease state was severe.

Patients with SFTS are prone to develop encephalopathy $[12,13]$. Cui et al. reported that $19 \%$ of patients with SFTS developed encephalopathy, and of these, the mortality rate was $44.7 \%$ [12]. In their study, early symptoms mainly included dimness of vision, bad feeling, cramp, sleepiness, and coma, which occurred within approximately 5 days after disease onset. Most risk factors for encephalopathy were consistent with the aforementioned factors related to 
a

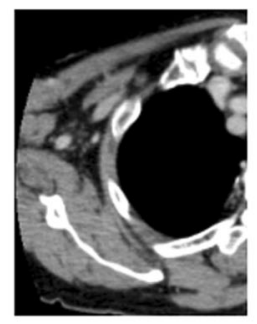

b

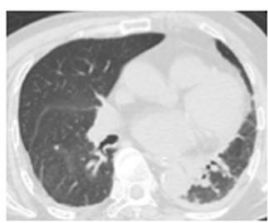

Day 1

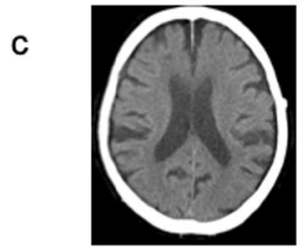

Day 1
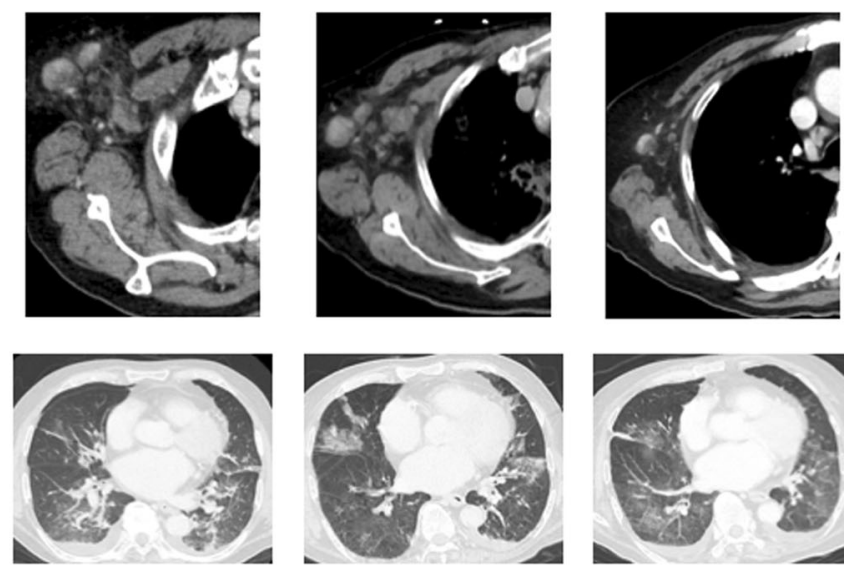

Day 9

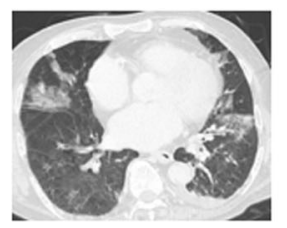

Day 21

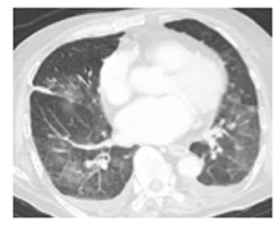

Day 51

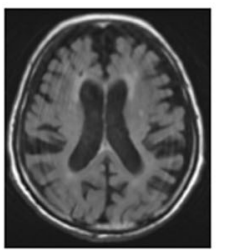

Day 63

Fig. 3 Chest and brain imaging. (a) Right axillary lymphadenopathy gradually reduced. (b) Chest CT showed diffuse ground glass opacity on days 9, 21, and 51. (c) Head CT (Day 1) and MRI (Day 63) showed no remarkable findings except cerebral atrophy

poor prognosis. In addition, Park et al. reported that CSF analysis exhibited glucose and protein levels within the normal range, an increase in cell count in the CSF was not remarkable, and the SFTSV genome was positive in $75 \%$ of cases [13]. These findings, along with the findings in this study, may be an important piece of information to advance our understanding of the pathophysiological mechanisms of encephalopathy in SFTS patients. Encephalopathy/encephalitis is a common complication of SFTS, and SFTSV-RNA is frequently detected, especially in the CSF of patients with symptoms or signs of central nervous system manifestations [13]. Although a positive PCR result for the SFTSV genome does not necessarily indicate infectivity of the virus unless culture isolation of live virus is not demonstrated, strict contact isolation precautions should be considered when performing CSF study in SFTS patients regardless of serum viral load.

In previous reports, SFTSV was detected not only in the blood and CSF but also in the urine, gastric juice, sputum, and semen $[9,14]$. A case in which viruses did not disappear after plasma exchange has also been reported [14]. Moreover, there were several case reports that strongly suggest droplet infection [15], including a case in which a patient with SFTS developed severe pneumonia in an early stage after disease onset, and two caregiving family members also developed SFTS [16]. In addition, there was a case report of SFTS development in two persons who did not touch the body fluid of an
SFTSV-infected corpse but stayed for a long period of time in a small room where the corpse was laid, indicating a possibility of aerosol transmission of SFTSV [17]. In the present case, we isolated the patient in a negativepressure airborne-isolation room from the time of the first examination and managed with droplet/contact isolation precautions. Specifically, we wore N95 masks, aprons, gloves, and face shields. In the intensive care unit, isolation with curtains and zoning were maintained to promote precaution. Although blood virus levels became undetectable on day 16, the virus genome had been persistently positive in sputum, and thus, the same countermeasures against infection were continuously conducted. Virus genomes in sputum remained positive until day 79. However, we confirmed the negativity on day 127 and subsequently removed countermeasures against droplet infection. Taken together, there might be cases in which SFTS virus might remain in various fluids and tissues even after it disappears from the blood. It may be possible that body fluids such as respiratory excretions become a source of infection; thus, careful management is needed.

In conclusion, we herein present an SFTS patient in whom encephalopathy, pneumonia, and invasive fungal infections developed. SFTSV-RNA was detected in the patient and the tick that bit this patient. Although the general condition improved and serum SFTSV levels reached a peak and decreased, a high level of SFTSV- 
RNA was detected in the CSF. Furthermore, the virus genome remained positive in the sputum for a long period of time, although viremia and pneumonia improved. These findings indicate that even when blood virus levels become undetectable, SFTSV may remain in other body fluids or tissues. Body fluids such as sputum can be a potential source of infection to others; thus, careful attention is necessary for countermeasures against SFTSV infection.

\section{Supplementary information}

Supplementary information accompanies this paper at https://doi.org/10. 1186/s12879-020-05012-8.

Additional file 1. Table 1. Laboratory data from the first examination

Additional file 2. Detection of SFTSV-RNA from a tick with qRT-PCR. Materials and methods for QRT-PCR and a figure showing amplification curves.

\section{Abbreviations}

BALF: Bronchoalveolar lavage fluid; CSF: Cerebrospinal fluid; CT: Computed tomography; PCR: Polymerase chain reaction; SFTS: Severe fever with thrombocytopenia syndrome; SFTSV: Severe fever with thrombocytopenia syndrome virus

\section{Acknowledgments \\ Not applicable.}

\section{Authors' contributions}

$\mathrm{KA}, \mathrm{TM}, \mathrm{KO}, \mathrm{AU} 1, \mathrm{HS}, \mathrm{MT}, \mathrm{TS}, \mathrm{MS1}, \mathrm{KY} 1, \mathrm{YI}$, and $\mathrm{KI}$ treated the patient and participated in the data acquisition and interpretation. TT, SK, KY2, and HM provided additional insights into the clinical course of the case and participated in the data interpretation. SS, KM, AU2, SM, TY, TK, MS2, and MS3 were involved in the detection of SFTSV, measurement of viral load, and interpretation of the data. KA, TM, and MS3 were major contributors in writing the manuscript. All authors read and approved the final manuscript.

\section{Funding}

This work was partially supported by grants from the Japan Agency for Medical Research and Development to M. Saijo (grant number JP18fk0108002). The financial supporter had no role in the study design, data collection and analysis, decision to publish, or preparation of the manuscript.

\section{Availability of data and materials}

The datasets used and/or analysed during the current study are available from the corresponding author on reasonable request.

\section{Ethics approval and consent to participate}

Not applicable.

\section{Consent for publication}

Written informed consent was obtained from the patient using the BioMed Central Consent Form for publication of this case report and any accompanying images. A copy of the written consent is available for review by the Editor of this journal.

\section{Competing interests}

The authors declare that they have no competing interests.

\section{Author details}

'Department of Respiratory Medicine, Nagasaki University Hospital, Nagasaki, Japan. ${ }^{2}$ Present Address: Department of Respiratory Medicine, Nagasaki Prefecture Shimabara Hospital, Nagasaki, Japan. ${ }^{3}$ Department of Infectious Diseases, Nagasaki University Graduate School of Biomedical Sciences, Nagasaki, Japan. ${ }^{4}$ Present Address: Department of Internal Medicine, Goto Central Hospital, Nagasaki, Japan. ${ }^{5}$ Present Address: Department of
Respiratory Medicine, Sasebo City General Hospital, Sasebo, Japan. ${ }^{6}$ Department of Virology, Institute of Tropical Medicine (NEKKEN), Nagasaki University, Nagasaki, Japan. ${ }^{7}$ Nagasaki University Infection Control and Education Centre, Nagasaki University Hospital, Nagasaki, Japan. ${ }^{8}$ Division of Intensive Care, Nagasaki University Hospital, Nagasaki, Japan. ${ }^{9}$ Department of Laboratory Medicine, Nagasaki University Graduate School of Biomedical Sciences, Nagasaki, Japan. ${ }^{10}$ Department of Veterinary Science, National Institute of Infectious Diseases, Tokyo, Japan. ${ }^{11}$ Department of Virology I, National Institute of Infectious Diseases, Tokyo, Japan.

Received: 27 November 2019 Accepted: 1 April 2020 Published online: 15 April 2020

\section{References}

1. Maes P, Adkins S, Alkhovsky SV, Avsic-Zupanc T, Ballinger MJ, Bente DA et al. Taxonomy of the order Bunyavirales: second update 2018. Arch Virol. 2019;164(3):927-41.

2. Fang LQ, Liu K, Li XL, Liang S, Yang Y, Yao HW, et al. Emerging tick-borne infections in mainland China: an increasing public health threat. Lancet Infect Dis. 2015;15(12):1467-79.

3. Saijo M. Pathophysiology of severe fever with thrombocytopenia syndrome and development of specific antiviral therapy. J Infect Chemother. 2018; 24(10):773-81.

4. Kato H, Yamagishi T, Shimada T, Matsui T, Shimojima M, Saijo M, et al. Epidemiological and clinical features of severe fever with thrombocytopenia syndrome in Japan, 2013-2014. PLoS One. 2016:11(10):e0165207.

5. Kim KH, Yi J, Kim G, Choi SJ, Jun Kl, Kim NH, et al. Severe fever with thrombocytopenia syndrome, South Korea, 2012. Emerg Infect Dis. 2013; 19(11):1892-4.

6. Takahashi T, Maeda K, Suzuki T, Ishido A, Shigeoka T, Tominaga T, et al. The first identification and retrospective study of severe fever with thrombocytopenia syndrome in Japan. J Infect Dis. 2014;209(6):816-27.

7. Yu XJ, Liang MF, Zhang SY, Liu Y, Li JD, Sun YL, et al. Fever with thrombocytopenia associated with a novel bunyavirus in China. N Engl J Med. 2011:364(16):1523-32.

8. Li H, Lu QB, Xing B, Zhang SF, Liu K, Du J, et al. Epidemiological and clinical features of laboratory-diagnosed severe fever with thrombocytopenia syndrome in China, 2011-17: a prospective observational study. Lancet Infect Dis. 2018;18(10):1127-37.

9. Koga S, Takazono T, Ando T, Hayasaka D, Tashiro M, Saijo T, et al. Severe fever with thrombocytopenia syndrome virus RNA in semen. Japan Emerg Infect Dis. 2019;25(11):2127-8.

10. Kawamori F, Shimazu Y, Sato H, Monma N, Ikegaya A, Yamamoto S, et al. Evaluation of diagnostic assay for rickettsioses using duplex real-time PCR in multiple laboratories in Japan. Jpn J Infect Dis. 2018;71(4):267-73.

11. Chen Y, Jia B, Liu Y, Huang R, Chen J, Wu C. Risk factors associated with fatality of severe fever with thrombocytopenia syndrome: a meta-analysis. Oncotarget. 2017:8(51):89119-29.

12. Cui N, Liu R, Lu QB, Wang LY, Qin SL, Yang ZD, et al. Severe fever with thrombocytopenia syndrome bunyavirus-related human encephalitis. J Inf Secur. 2015;70(1):52-9.

13. Park SY, Kwon JS, Kim JY, Kim SM, Jang YR, Kim MC, et al. Severe fever with thrombocytopenia syndrome-associated encephalopathy/encephalitis. Clin Microbiol Infect. 2018;24(4):432.e1-432.e4.

14. Jeong EJ, Song JY, Lim CS, Lee I, Park MS, Choi MJ, et al. Viral shedding from diverse body fluids in a patient with severe fever with thrombocytopenia syndrome. J Clin Virol. 2016;80:33-5.

15. Moon J, Lee H, Jeon JH, Kwon Y, Kim H, Wang EB, et al. Aerosol transmission of severe fever with thrombocytopenia syndrome virus during resuscitation. Infect Control Hosp Epidemiol. 2018:1-4.

16. Zhu Y, Wu H, Gao J, Zhou X, Zhu R, Zhang C, et al. Two confirmed cases of severe fever with thrombocytopenia syndrome with pneumonia: implication for a family cluster in East China. BMC Infect Dis. 2017;17(1):537.

17. Gong Z, Gu S, Zhang Y, Sun J, Wu X, Ling F, et al. Probable aerosol transmission of severe fever with thrombocytopenia syndrome virus in southeastern China. Clin Microbiol Infect. 2015:21(12):1115-20.

\section{Publisher's Note}

Springer Nature remains neutral with regard to jurisdictional claims in published maps and institutional affiliations. 